\title{
Emily Aubert y Catherine Morland: Naturaleza e interiores en su camino hacia la madurez
}

\author{
María José Montes González \\ (Universidad de Santiago de Compostela)
}

\section{RESUMEN:}

La naturaleza y el ser humano han tenido, desde el principio de los tiempos, una estrecha relación. No cabe el entendimiento de una sin la otra. En el siguiente artículo se tratará de reflejar esa unión en relación a la mujer en dos novelas: The Mysteries of Udolpho de Anne Radcliffe y Northanger Abbey de Jane Austen. Si bien ambas desarrollan el proceso de madurez en la mujer y cómo la naturaleza e interiores interceden en este, la primera difiere de la segunda en presentar a una protagonista que es un dechado de virtudes y estereotipo de heroína. Tendrá que sortear multitud de trabas, tanto sociales como emocionales para alcanzar su plenitud como persona. Austen, por su parte, muestra el mismo proceso pero eligiendo como personaje principal a una joven que podría ser calificada como antiheroína. Ambas escritoras consiguen su meta: libertad emocional y madurez para sus protagonistas.

Palabras clave: Anne Radcliffe, Jane Austen, naturaleza, análisis comparativo

\section{ABSTRACT:}

Nature and human being have been, since the origin of the world, closely related. An understanding of them cannot be achieved separately. The following article will try to show this relation between women and nature in both novels: Anne Radcliffe's The Mysteries of Udolpho and Jane Austen's Northanger Abbey. It is true that these novels show the path to follow to reach female maturity and the role nature and interior settings have on it. One of the differences the reader may find is the chose female character: Radcliffe's heroine versus Austen's anti - heroine. The first one will have to cope with difficult situations from an emotional and social point of view to reach maturity. Austen shows the same process but, ironically, she chooses a female who is not considered a sterotype. Both writers get their goal: a complete woman that enjoys her emotional and personal freedom.

Keywords: Anne Radcliffe, Jane Austen, nature, comparative analysis

Austen parodia la novela de Anne Radcliffe The Mysteries of Udolpho en su novela Northanger Abbey. En el siguiente artículo, disertaré sobre las similiyitudes y diferencias que se pueden encontrar en ambas protagonistas. Tanto Radcliffe como Austen eligen un personaje femenino para mostrar el crecimiento personal, por antonomasia, hasta alcanzar la madurez. Tanto la familia como las circunstancias sociales obligan a Emily, en el caso de Udolpho, y a Catherine, en el de Nortanger, a crecer.

No se puede establecer una comparación entre estos dos personajes sin tener en cuenta que Emily Aubert es considerada como el estereotipo de heroína Gótica mientras que Catherine, la protagonista personaje principal de la novela de Austen, representa a la antiheroína por excelencia. A pesar de ser bastante distintas, ambas son elegidas por las novelistas para convertirse en heroínas sin tener en cuenta ni su status social ni la fortuna de sus familias.

Emily es una víctima de la sociedad patriarcal. En esta, todo está bajo control masculino y la prohibición reina en relación a la mujer. La inocencia es un punto clave en el crecimiento de Emily dado que ella no conoce el alcance de la maldad en el ser humano. Su obediencia y servicio para con su familia, especialmente su padre, son piezas clave en su crecimiento para poder alcanzar la madurez personal. Emily goza de una magnífica educación tal y como solía suceder en las mujeres de clase media alta; sin embargo desconoce cómo actuar por ella misma, sin apoyos, ni afrontar situaciones difíciles en la vida. Tras el fallecimiento de su padre, se abra ante ella el camino para su crecimiento y llegar a ser una mujer madura y plena. Tanto Radcliffe como Austen muestran personajes femeninos totalmente aislados socialmente y sin ningún tipo de conocimiento salvo aquel que corresponde al de ser mujer. Tanto su educación, como relaciones sociales y fortuna están supervisadas y tuteladas por la figura masculina, bien sea el padre o el marido. Cualquier atisbo de libertad o de llegar a ser una mujer culta e independiente se ve truncado por el mundo masculino en el que viven. La presión de Emily aumentará en el momento en el que llegue a los Alpes italianos dado al comportamiento de Montoni, el marido de su tía, con Emily. Para poder comparar a Emily con Catherine hay que destacar que es la naturaleza la encargada de enseñar a Emily en lo concerniente a la vida y a ella misma:

Emily wished to trip along the turf, so green and bright, with dew and to taste the full delight of that liberty, which the hazard seemed to enjoy as he bounded along the brow of the cliffs ... "Here is the real ingenuousness and ardour of youth!" he said to himself. "This young man has never been in Paris". 1

En el caso de Catherine será la sociedad quien realice esa función:

In society, marriage was recommended as an alliance of sense. Too much sensibility - still more, sensuality - was suspected; giddy romance would wither 
on stony soil ... Once married, a lady in polite society had four cardinal functions. The first duty was to obey her husband. Second, she had to produce heirs ... The third duty of the married lady was to run the household ... The fourth duty was to be ladylike, an ambassadress of grace. $\underline{2}$

Emily vivirá rodeada de montañas abruptas y caminos tortuosos que tienen como fin el castillo de Udolpho. Este paisaje representa sus inquietudes, miedos y desconocimiento respecto a su futuro. Tendrá que tomar decisiones y aprender a ser más fuerte algo a lo que ella no está habituada. Expuesto esto, el paisaje es un amigo que semeja lo contrario al comienzo de su travesía. Es este el que ayuda a Emily a ser cuidadosa y precavida a la hora de tomar una decisión y ser ella dueña y señora de sus actos. La protagonista no ha estado jamás sola en tales circunstancias ni aislada socialmente como lo está ahora. Esta situación es necesaria para su crecimiento. Su único amigo y confidente es el paisaje, el bosque frondoso en el que ella busca cualquier tipo de ayuda que le alivie la cautividad que está sobrellevando. Se podría comparar el castillo en el que Emily está viviendo con la sociedad masculina en la que las mujeres habitan. Emily es un ejemplo de todas las mujeres forzadas a vivir en una sociedad hostil para ellas. Radcliffe aísla a la heroína con el fin de que esta adquiera un mejor conocimiento de ella misma. El castillo tiene una doble función ya que, por una banda representa los sentimientos y miedos que Emily tiene que afrontar para conseguir su objetivo: ser una mujer madura y segura de sí misma. Por la otra, puede ser entendido el castillo como una metáfora de la sociedad patriarcal. Tal y como Hellen Mores cita en Gothic Forms of Feminine Fictions:

In the power of villains, (Radcliffe's) heroines are forced to do what they could never do alone, whatever their ambitions: scurry up to the top of pasteboard Alps, spy out exotic vistas, and penetrate bandit-infested forests. And indoors, inside Mrs. Radcliffe's castles, her heroines can shuttle miles along corridors, descend into dungeons, and explore secret chambers without a chaperone, because the Gothic castle, however much in ruins, is still an indoor and therefore freely female space. ${ }^{3}$

Se puede pensar que el interior del castillo es un contexto libre para la mujer desde el punto y hora en que la protagonista puede explorar por donde quiera. El castillo, solitario y frío, es un reflejo de los sentimiento de Emily. Este "uncanny sentiment" causa repulsión en ella pero, al mismo tiempo, se convierte en una atracción difícil de resistir. El interior del castillo es una metáfora de la mente de Emily:

All without was silent and dark, unless that could be called light, which was only the glimmer of the stars ... The fierce and terrible passions, too, which so often agitated the inhabitants of this edifice, seemed now and hushed in sleep; ... Emily's heart was not so; but her sufferings, though deep, partook of the gentle character of her mind. 4
Ella nunca ha estado en una vivienda como esa y su corazón alberga sentimientos contradictorios. Al descubrir pasillos, sótanos desconocidos y la humedad del ambiente, Emily puede escuchar a lo lejos voces desconocidas. La protagonista presta atención a esos lugares ocultos, a desconocidos e inquietantes pasos que se escuchan y forman parte ya de su vida y de su corazón. Un nuevo mundo se abre ante ella. El cambio se produce no sólo a nivel físico sino también psicológico. Su aislamiento de la casa paterna era imprescindible. Un mundo externo e interno se presenta ante ella con la finalidad de que lo descubra y aproveche.

La relación entre el alter ego de Emily, la naturaleza y el castillo se expresa en su estancia en el castillo. Esta relación limpia su corazón y alma de conceptos erróneos y prohibiciones paternas inútiles que impiden su crecimiento. La abadía de Saint Agnes es otro pilar puesto que el estar en contacto con las monjas le anima a convertirse en una mujer valiente. Es curioso como Radcliffe utiliza la abadía como lugar sagrado en el que se revela la verdad sobre Lady Laurentini. Incluso lugares considerados como sagrados no están exentos de maldad. Emily descubre la abadía como lugar en el que puede encontrar refugio pero también puede acoger a aquellos que cometieron acciones horrendas. La novela de Radcliffe termina con el regreso de Emily a casa con su amado, Valancourt. El viaje podría entenderse como una travesía vital en la que una inocente y dulce Emily regresa a La Vellé de Udolpho; en esta ocasión vuelve a casa como una mujer madura, independiente y libre que ha aprendido a dominar sus impulsos y temores. Su proceso de crecimiento y su inocencia pertenecen ahora al pasado. No es la joven inexpresiva, superficial o hipócrita criticada por Radcliffe y Austen. Emily se ha transformado en una mujer sensata y con el suficiente conocimiento de sí misma para no dejarse ser una mascota del patriarcado. La heroína alcaza su culmen una vez se libera de sus temores y limitaciones.

Catherine, en Northanger Abbey, es un ejemplo claro de lo que una autora considera como una mujer coherente y consecuente. El primer aspecto que difiere entre Emily y Catherine es el hecho de que Emily se presenta al lector como un dechado de virtudes. Es el prototipo de mujer y heroína; una mujer masculina en tanto en cuanto afronta las dificultades de la vida de manera racional, sin dejarse llevar por sensiblerías. Catherine, por su parte, no es virtuosa en ninguna de las tareas que se suponen esenciales en la formación de una mujer tales como: pintura, costura o música. Su masculinización se puede observar en su comportamiento cuando juega con sus hermanos, es incluso hombruna, y disfruta plenamente en contacto con la naturaleza. La educación de ambas protagonistas ha sido muy diferente. Emily pertenece a una clase social superior a la de Catherine y el destino de la primera es el de llegar a ser buena esposa tras haber sido una hija ejemplar. Emily debe aprender a ser sensata y reservada tal y como las reglas sociales han dictaminado que debe ser una mujer. Catherine has crecido en un ambiente familiar mucho más relajado y con menos ataduras a las normas sociales. La educación de Catherine es casi idéntica a la de Emily salvo en la rigurosidad de la misma dado que Catherine no es tan paciente y dócil como Emily. La madre de Catherine trata de educarla pero sus intenciones se ven frustradas en multitud de ocasiones. Gracias a lo que se podría 
denominar antiheroísmo en el caso de Catherine, es esta característica la que la convierte en heroína. A través de este personaje, Austen recurre a la ironía para criticar, no sólo la figura de la heroína en la novela Gótica sino también la imagen de mujer que Radcliffe presenta en sus novelas. Es a través de la ironía y la sátira cómo Austen muestra el prototipo de mujer que vivía en el siglo XVIII y XIX. La mujer no es buena en nada y es ese hecho parte fundamental de su educación. La inocencia y dulzura de Catherine son dos impedimentos que obstaculizan su ser consciente de las dificultades de la vida. Es inexperta y el viaje de Catherine es a Bath, en el mismo país en le que vive, mientras que Emily viaja al extranjero. No obstante, no es de extrema importancia que Catherine deba abandonar el país para alcanzar la madurez. En Bath, se encuentra lo suficientemente lejos de casa para sentir la soledad e individualismo que reina en la ciudad siendo estos dos aspectos la base de su crecimiento personal. Dado que Catherine no ha crecido en un ambiente sofisticado, no es consciente de lo hipócrita y superficial que puede llegar a ser la sociedad en Bath. Emily, no solo está sola en Udolpho salvo con la compañía de una sirvienta cuya fantasía es desmedida sino que también lo está geográficamente de su casa natal. Udolpho carece de vecinos y la naturaleza que lo rodea podría admitir cualquier adjetivo salvo armoniosa. Catherine, por su parte, está acompañada prácticamente todo el tiempo a lo largo de la novela. Sus momentos a solas en la abadía de Northanger son primordiales para la tarea que se ha impuesto: descubrir la causa de la muerte de Mrs Tinley y percatarse del mayúsculo error que ha cometido al pensar que $\mathrm{Mr}$ Tinley había sido partícipe en la muerte de su esposa. Un importante hecho es el de la multitud de gente que rodea a Catherine representando, esta, el mundo material, superficial y patriarcal en que Catherine vive. Es esta atmósfera la que la absorbe y subyuga sin darle oportunidad para que piense por sí misma y correctamente. Esto se alcanza cuando está sola y se establece una distancia entre ella y la sociedad en la que vive.

El punto débil de Catherine es su obsesión con las novelas Góticas. Estas funcionan como vía de escape; un mundo al que ella pertenece puesto que es consciente que difiere, en la forma de ser, de sus compañeras. Es en las novelas Góticas y en sus heroínas en las que Catherine encuentra un referente. Su deseo oculto es el de vivir una vida tan apasionada como el de las protagonistas de sus novelas. Catherine siente tal devoción por este tipo de lecturas que cuanto más obsesionada está con ellas, más inmadura parece. No es capaz de establecer una diferencia entre ficción y realidad. Sólo anhela la libertad y sentirse libre de la sociedad opresora en la que vive. Sin embargo, Austen critica el excesivo uso de la imaginación. Esta es una de las principales diferencias entre las dos novelas. Mientras que Catherine es el personaje principal de una novela Realista, Emily lo es de un Romance. Austen critica la excesiva imaginación y fantasía de Catherine. Emily vive en mundo son matices fantásticos que Austen censura de manera velada mediante Catherine.

Catherine, al igual que Emily, goza de una estrecha relación con la naturaleza e interiores a pesar de no vincularse tan estrechamente con estos elementos. No se encuentran, en Northanger Abbey, descripciones de paisajes ni su efecto en la heroína. Cierto es, no obstante, que cuando
Catherine visita la abadía no deambula por los pasillos ni está acompañada por sombras amenazantes. Si su cambio personal comienza una vez se encuentra la abadía, el de Emily tiene lugar en el castillo. Con la desbocada imaginación en funcionamiento y con las novelas de Radcliffe como referencia, Catherine anhela vivir una vida como la de Emily. Sin embargo, según van pasando los días, Catherine caerá en la cuenta de las diferencias existentes entre su vida y la de la heroína de Udolpho. La vida de Catherine ni se semeja a la de Emily ni comparte el ámbito social. La antiheroína de Austen experimentará su transformación personal en un contexto totalmente distinto al soñado. En ambas novelas, el edificio Gótico (la abadía y el castillo) representan los miedos y fantasías de las protagonistas. Emily recorre el castillo con el fin de encontrar una solución a lo que le está sucediendo durante su estancia en él y Catherine busca una pista, en la galería prohibida, que le ayude a desvelar la causa de la muerte de Mrs. Tinley. Ambas mujeres tienen el paso vetado a determinados lugares; en el caso de Emily, es Montoni quien ejerce esa prohibición y Mr Tinley en el de Catherine. No obstante, el deseo por conocer la verdad es más fuerte en estas mujeres que cualquier obstáculo; con lo cual será esa necesidad por saber lo que las impulse a seguir adelante. Catherine recorre la galería intentando averiguar el motivo del fallecimiento de Mrs Tinley, como se ha expuesto previamente, pero, al mismo tiempo, recorre su yo interior para un mayor conocimiento de su alter ego. Catherine y Emily llevan sobre sus hombros una gran carga social y patriarcal. No son libres para actuar por ellas mismas ni para poder conocerse mejor. En cuando surge la más mínima oportunidad, se aferran a ella. La abadía es el lugar elegido para el cambio personal que sufrirá Catherine y donde recapacitará sobre su comportamiento infantil hasta el momento y la perniciosa influencia que las novelas Góticas han ejercido en ella. Se avergonzará de sí misma y esto supondrá el primer paso hacia la madurez.

Tanto la oscuridad como la noche, las formas generadas por las sombras, el tiempo intempestivo y el miedo recorriendo sus venas son experiencias que Catherine y Emily sienten cuando caminan por los pasillos secretos en sus hogares provisionales. Es cierto que Emily encuentra el retrato de una dama tras un velo negro; Austen, recurriendo a la ironía, trata de hacer vivir a Catherine una situación similar cuando en su afán por tener una vida llena de aventuras, esta confunde el hallazgo de unos recibos de lavandería con unos documentos importantes. Ambos descubrimientos son importantes tanto para Emily como Catherine ya que enseñan a las protagonistas lo engañosa y poco fiable que puede llegar a ser la imaginación. Northanger está decorada con un estilo moderno y exótico, metáfora de la viva imaginación de Catherine ya que no se trata de un personaje plano, sin riqueza interior. Catherine es compleja; aprendemos de ella gracias a sus pensamientos y temores. Su fantasía se convierte en su virtud y su defecto. La imaginación de Emily es rica también puesto que la guía lejos de la realidad. Sin embargo, la conducirá a cometer errores y aprender cómo es el mundo realmente. Catherine y Emily van más allá del mundo en el que viven y, una vez allí, disfrutan d un conocimiento prohibido por el hombre hasta ese momento.

Catherine se siente aislada cuando está en los salones de baile o de té. Lo mismo le sucede a

URL: http://oceanide. netne. net/articulos/art1-1.php \# 
Emily cuando está en Venecia. Allí, la sociedad es tan snob y superficial que Emily no encaja en ella. Tanto Emily como Catherine no han crecido en ese ambiente $y$, en consecuencia, les resulta desconocido y hostil. No encuentran su sitio en esa sociedad. Tras haber estado en la abadía, Catherine regresa a casa como una mujer plena y con sus propios principios. La misma situación se plantea en el caso de Emily quién vuelve a La Vallée tras su paso por Udolpho. El regreso a casa implica la alcanzada madurez personal. Ambas mujeres cierran el círculo de su viaje tras haber superado sus miedos y errores. Radcliffe y Austen destacan las dificultades que cualquier mujer, heroína o no, debe superar para ser consciente de su valía dentro de la sociedad y reconocer su riqueza interior que la llevará a la madurez.

Como puede apreciarse al leer las novelas, los personajes masculinos son importantes en la vida de Emily y Catherine. Aunque Valancourt, el pretendiente de Emily, es un hombre con muchos fallos, aguarda y vela por ella cuando no está de manera presencial. Henry Tinley es el tutor de Catherine; la trata con cariño y paciencia ya que es consciente de que no es una mujer madura. Austen recurre al personaje masculino como medio para ayudar a la mujer a conocerse mejor mostrando, tal vez, su aceptación, hasta cierto punto, del sistema patriarcal imperante en Inglaterra en aquella época.

Si Emily y Catherine son la representación masculina de la mujer, Henry Tinley y Valancourt son la parte femenina. Ambos no poseen un carácter fuerte. Valancourt, tal y como se ha explicado previamente, pasa sus días viajando de pueblo en pueblo y viviendo del dinero que su hermano mayor le administra. Cuando está en Venecia, apuesta en juegos de azar perdiendo dinero y es incapaz de encontrar una alternativa para liberar a Emily del cautiverio de Montoni. El punto final se alcanza cuando Emily accede a casarse con él pero, una vez más, la falta de decisión de Valancourt hace su aparición: tendrán que vivir en casa de Emily y con el dinero que ella posee por su herencia como único ingreso. Valancourt no intenta mejorar esta situación. En este caso es la mujer la que asume el mando y el papel masculino puesto que es Valancourt quien está bajo la protección de Emily y no a la inversa. Henry Tinley podría ser considerado como un hombre femenino desde el momento en que es a Catherine a quien le gusta revolcarse por la hierba sin preocuparse si se ensucia o no. A Henry le encantan las novelas Góticas que son consideradas como manjar para el público femenino. Es un experto en ropa y moda. Muestra su valor cuando se enfrenta a su padre tras haber decidido este enviarla de vuelta a casa desde Northanger al descubrir que no posee riquezas. Henry Tinley y Valancourt son hombres femeninos aunque Tinley de una manera superficial ya que demuestra su arrojo al enfrentarse a la figura paterna y hacerse oír.

El contexto exterior e interior desvelan el verdadero yo de Emily y Catherine. Este marco presenta los miedos y carencia de conocimientos de ambas y, como colofón, les conceden la felicidad y plenitud. La verdadera diferencia entre ellas radica, sin embargo, en su función en la novela. Emily es una joven inocente rodeada de maldad externa y ajena a ella mientras que Catherine es una parodia de este tipo de heroína. Ambas descubren quienes son pero de manera distinta, estas distinciones determinan el tipo de novela ya que Radcliffe presenta a una heroína Romántica y Austen a una antiheroína Realista.

\section{NOTAS AL PIE}

${ }^{1}$ Anne Radcliffe. The Mysteries of Udolpho. Oxford: Ed. Bonamy Dobreé: Oxford University Press,

1983, p 36.

${ }^{2}$ Jane Austen. Northanger Abbey. London: Penguin Popular Classics, 1984, p 57.

${ }^{3}$ Susan Becker. Gothic Forms of Feminine Fictions. Manchester: Manchester University Press, 1999, p. 8 - 9

${ }^{4}$ Anne Radcliffe. The Mysteries of Udolpho. Oxford: Ed. Bonamy Dobreé: Oxford University Press, 1983, p. 329.

\section{REFERENCI AS BI BLI OGRÁFICAS}

- AUSTEN, Jane. (1984). Northanger

Abbey. London: Penguin Popular Classics.

- BARRET, Eaton. (1928). The Heroine. New York:

Frederick A. Stokes.

- BOTTING, Fred. (1996). Gothic. London:

Routledge.

- CARTER, Margaret. (1986). Specter or Delusion? The Supernatural in Gothic Fiction. London: U.M.I. Research Press.

- FERGUSON ELLIS, Kate. (1989). The Contested Castle. Gothic Novels and The Subversion of Domestic Ideology. Illinois: University of Illinois. - FREUD, Sigmund. (1985). "The Uncanny" or On Creativity and the Unconscious papers on the philosophy of Art, Literature, Love, Religion. New York: Harper \& Row.

- GALPERIN, William H. (2003). The Historical Austen. Philadelphia: Pennsylvania: University of Pennsylvania Press.

- GILBERT, Susan and GUBAR, Susan. (1979). The Madwoman in the Attic. New Haven: Yale University Press.

- GONZÁLEZ MORENO, Beatriz. (2007). Lo

Sublime, Lo Gótico y Lo Romántico: La experiencia estética en el romanticismo inglés. Cuenca: Ediciones de la Universidad de Castilla - La Mancha.

---. (2007). "Gothic Excess and Aesthetic Ambiguity in Charlotte Dacre's Zofloya". Women's Writing.Volume 14, 3. $419-434$.

- GREENBLATT, Stephen. (1980). Renaissance Self - Fashioning: From More to Shakespeare. Chicago and London: University of Chicago Press.

- HEILAND, Donna. (2004). Gothic \& Gender. An

Introduction. Oxford: U.K: Blackwell Publishing. - HOEVELER, Dianne L. (1997). Gothic Feminism: The Professionalization of Gender from Charlotte Smith to the Bröntes. Pennsylvania: The Pennsylvania State University Press.

- HOWARD, Jacqueline. (1994). Reading Gothic

Fiction. New York: Oxford University Press.

- HUTCHEON, Linda. (1985). A Theory of Parody:

The Teachings of Twentieth - Century Art Forms. New York: Methuen

- KILGOUR, Maggie. (1995). The Rise of the Gothic Novel. London: Routledge. - LE FAYE, Deidre. (2002).J ane Austen. The World of Novels. London: Frances Lincoln Limited.

- LEWIS, Paul. (1983). Gothic and Mock Gothic: The Repudiation of Fantasy in Barret's Heroine. 
English Language Notes, 21/1 (sept.1983), p. 44.

- MILES, Robert. (2001). A Companion to the

Gothic. Anne Radcliffe and Mathew Lewis. Oxford:

Blackwell Publishing Ltd.

- MOERS, Ellen. (1979). Literary Women. London:

The Women's Press, 1979.

- MORRIS, David B. (1985). "Gothic

Sublimity". New Literary History 16:2. 299 - 319.

- MURRAY, Venetia. (1999). High Society in the

Regency Period. London: Penguin Books.

- NAPIER, Elizabeth. (1987). The Failure of the

Gothic. Oxford: Clarendon Press.

- PORTER, Roy. (1991). English Society in the

Eighteen - Century. Harmondsworth, Middlesex:

England. Penguin Books.

- RADCLIFFE, Anne. (1986). The Mysteries of

Udolpho. Ed. Bonamy Dobreé. Oxford: Oxford University Press.

- SIXTO REY, Teresa. Breaking Stereotypes: Wide Sargasso Sea and the Humanisation of Bertha Mason.(Trabajo Inédito).

Title: Emily Aubert and Catherine Morland: Outdoors and indoors in their way to maturity. 